\title{
The barrel cortex-integrating molecular, cellular and systems physiology
}

Received: 18 July 2003 / Accepted: 5 August 2003 / Published online: 19 September 2003

(C) Springer-Verlag 2003

\begin{abstract}
A challenge for neurobiology is to integrate information across many levels of research, ranging from behaviour and neuronal networks to cells and molecules. The rodent whisker signalling pathway to the primary somatosensory neocortex with its remarkable somatotopic barrel map is emerging as a key system for such integrative studies.
\end{abstract}

Keywords Neocortex - Somatosensory cortex · Whiskers · Behaviour - Plasticity - Neuronal networks · Sensory response $\cdot$ Synaptic mechanisms

\section{Introduction}

The aim of modern integrative neurophysiology is to understand the neural basis of behaviour at the neuronal network, the cellular and the molecular level. Increasingly, the rodent has become the experimental subject of choice, providing complex and interesting behaviour and allowing full use of molecular and genetic interventions. There has been rapid, recent technological progress in the study of cellular and molecular neurophysiology of rodents allowing the study of networks of genes and neurons. To integrate information across many levels of research requires a well-defined system that can be analysed quantitatively. The rodent barrel cortex and the signalling pathway leading to it have many attractive features, perhaps most importantly a clearly defined anatomical map corresponding to its functional layout. Study of this sensory pathway may ultimately allow detailed understanding at the molecular level of how the activity of neurons and their synaptic connections generate sensory perceptions leading to behaviour.

C. C. H. Petersen $(\bowtie)$

Laboratory of Sensory Processing, Brain and Mind Institute,

SV-INS-LSENS, AAB105, EPFL,

1015 Lausanne, Switzerland

e-mail: carl.petersen@epfl.ch

Tel.: +41-21-6938352

Fax: +41-21-6938353

\section{Behavioural analysis of whisker sensation}

Rodents are nocturnal animals often living underground in tunnels. Under many natural circumstances they must gather information concerning their surroundings without use of vision. It is presumably for this reason that rodents have evolved a highly sensitive array of whiskers on their snouts (Fig. 1A). These vibrissae are thin flexible hairs and their base is surrounded by sensory nerve endings that detect whisker motion. The whiskers are arranged in a highly stereotypical pattern allowing the identification of individual whiskers (Fig. 1B). The most posterior of these whiskers protrude a few centimetres from the snout, whilst anteriorly, around the lips, they are only a few millimetres long. The different whiskers most probably serve different functions, with the short anterior vibrissae gathering primarily textural information from an object located close to the mouth, whereas the large whiskers gather spatial information regarding the location of objects surrounding the head (Brecht et al. 1997). Whichever whiskers are used, the acquisition of sensory information is often a highly active process, either involving head movements in the case of the short vibrissae or the direct movement of the whiskers through muscles in the whisker pad (Dorfl 1982; Berg and Kleinfeld 2003). Indeed, as a rodent approaches an object of interest, it moves the posterior whiskers back and forth rhythmically (Fig. 1C) at around $10 \mathrm{~Hz}$ in a behaviour known as whisking (Welker 1964). This process of moving the whiskers across an object is likely to enhance sensitivity, in the same way as moving our fingertips across an object allows us to detect fine textures that a stationary finger simply cannot feel. The neural control of whisking and its effects on sensory pathways have begun to be studied. Whisking is likely to be regulated by a brainstem central pattern generator (Gao et al. 2001) controlling the trigeminal facial motoneurons, which innervate the muscles that move the whiskers. In addition, these circuits are subject to higher-level control from the motor cortex (Donoghue and Wise 1982; Miyashita et al. 1994; Hattox et al. 2002) that probably contributes to the complex whisker movements evoked 

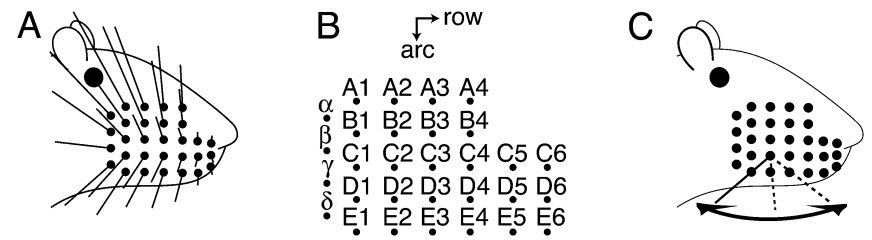

D texture discrimination

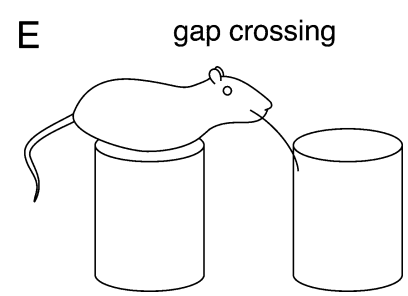

Fig. 1A-E Pattern and function of rodent whiskers. A The snout of the rodent is surrounded by an impressive array of whiskers laid out in a stereotypical pattern. At the base of each whisker the follicle is surrounded by sensory nerve endings that detect whisker movements. B The pattern of whiskers on the rodent snout, especially amongst the large posterior whiskers are easy to recognize and a standard nomenclature has been developed to describe each individual whisker. The whiskers are laid out in rows (labelled $A-$ $E$ ) running along the anterior-posterior axis. The arcs (labelled 1,2, $3 . .$.$) are oriented orthogonally. \mathbf{C}$ The whiskers can be moved by muscles either individually or, more commonly, altogether in synchrony. Acquisition of sensory information can thus be a highly active process, and indeed as a rodent approaches an object of interest it will often move the whiskers back and forth at high frequency along the row-like orientation. Such whisking behaviour may increase sensitivity to textural features. D Rodents can be trained to discriminate textures, behaving differently according to the texture presented. The sensitivity of the whisker system matches that of the primate finger tip. E Rodents can be trained to judge the distance between two platforms using whisker-related sensory input and only to cross if from one platform to the other if the whisker makes contact with the other platform

during active acquisition of sensory information upon contacting an object of interest. There is significant cortical action potential activity in the primary somatosensory barrel cortex resulting from whisker movements, even during free whisking when the whiskers do not contact an object (Fee et al. 1997). Neural activity associated with detection of texture or object location thus results from an interaction with the activity generated by the whisking itself.

A number of tasks have been developed to study whisker-dependent behaviour quantitatively. Carvell and Simons (1990) have trained rats to discriminate different textures using only the whiskers (Fig. 1D). Their psychophysical measurements indicate a comparable sensitivity to the primate fingertip. Interestingly, the ability to make fine discrimination of textures is dependent upon the animal experiencing normal whisker sensation early in life, since it can be disrupted by infantile whisker trimming (Carvell and Simons 1996). Recently, Prigg et al. (2002) have extended these behavioural studies to combine automated presentation of textures, video recording of behaviour and extracellular electrophysiological recordings.

Another behavioural task, first described by Hutson and Masterton (1986), that has been further extensively studied is gap crossing (Fig. 1E). In this test the rodent is placed on an elevated platform from which it can cross to another platform some distance away. The rodent is trained to reach out with its whiskers across the gap separating the platforms and, if it locates the target platform with a whisker touch, it will then jump across to receive its reward. Even animals with only a single intact whisker (all others having been trimmed) can perform this task. Mathew Diamond and co-workers have used this task to study the spatial distribution of tactile learning (Harris et al. 1999). After training an animal to perform the gap crossing task with one whisker, they cut the whisker off and then reattached it to the stub of one of the other previously trimmed whiskers. They found that the closer the whisker was reattached to its original location the quicker the animal relearned to perform the gap-crossing task, which correlated remarkably well with the spatial extent of single whisker-evoked extracellularly-recorded action potential activity in primary somatosensory barrel cortex. This raises the possibility that the learning of this whisker-related task might occur through neural changes in the barrel cortex localized to the representation of the whisker that was used to learn the task.

Some tasks, however, can only be performed when many whiskers are present. Nicolelis and colleagues have demonstrated that as whiskers are removed from the array there is a gradual loss of ability to discriminate with respect to the width of a large aperture (Krupa et al. 2001). In this test whiskers on both left and right sides of the snout contact the aperture simultaneously and it is likely that the correlated bilateral activity of both left and right barrel cortices are involved in this sensory perception task. Interestingly, this aperture-width-discrimination task is performed without whisking (although with head movements) and cutting the facial motor nerve (which eliminates whisker movements) has no effect on the ability of rats to discriminate.

Having defined whisker-dependent behaviours, the next crucial experimental step is of course to relate this with brain activity and genetic manipulations. The first steps in this process, namely the analysis of this somatosensory pathway at the molecular and cellular level, have provided interesting data.

\section{The sensory signalling pathway from whisker to cortex}

Important aspects of the sensory signalling pathway from whisker to cortex (Fig. 2) have been defined at both the anatomical and functional level. The whisker hair follicles are surrounded by infraorbital nerve endings of trigeminal sensory neurons, which directly detect movement of the vibrissa. Each hair follicle is innervated by 50-200 sensory neurons, depending on the location of the whisker within the mystacial pad, with the large posterior whiskers being the most densely innervated (Welker and Van der Loos 1986). These sensory neurons have their cell bodies located in the trigeminal ganglion and project to the brainstem. An individual sensory neuron responds with 


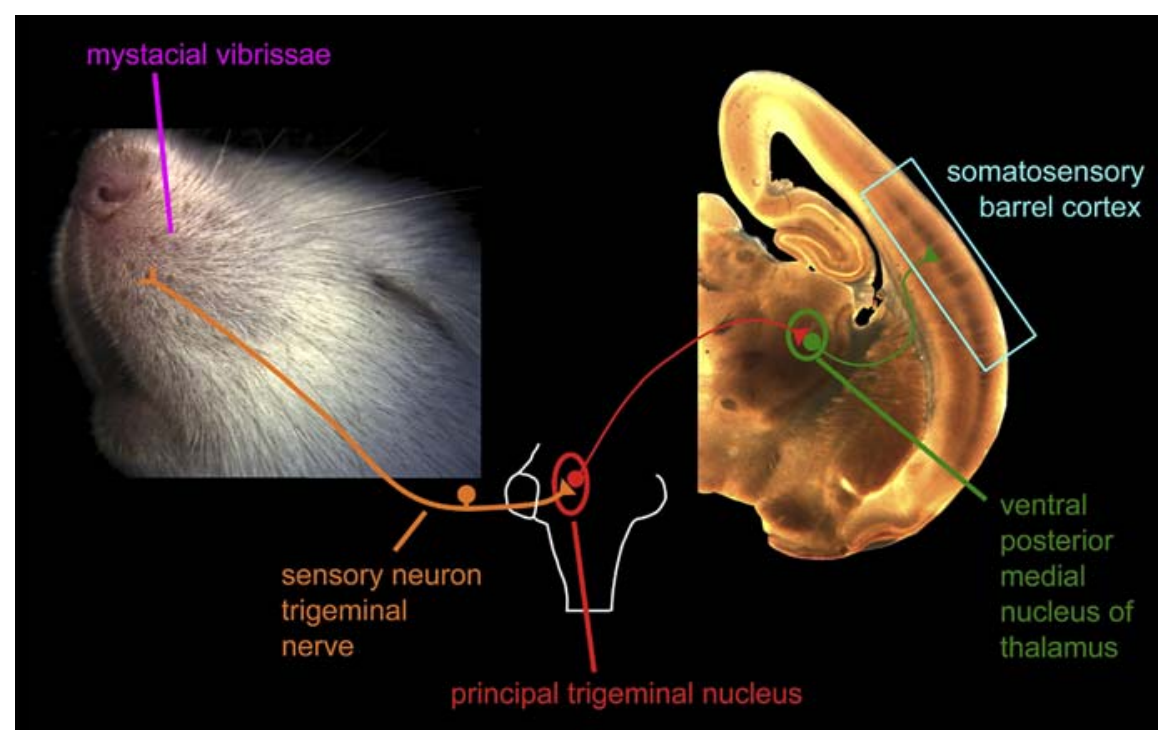

Fig. 2 The primary signalling pathway from whisker to cortex. Sensory neurons of the trigeminal ganglion have nerve endings in the whisker pad. These neurons fire action potentials in response to whisker movement, which release glutamate at brainstem synapses. Neurons in the principal trigeminal nucleus are the main recipients of this excitation. The neurons and their inputs are clustered as barrelettes forming a well-ordered map of the sensory periphery.
These brainstem neurons project to the ventral posterior medial thalamus (VPM), where their arborizations again cluster into units termed barreloids. The VPM barreloid neurons project to primary somatosensory cortex, where they terminate in somatotopically arranged clusters in layer 4 forming barrels. The layer 4 barrel neurons thus form the first layer of cortical processing of sensory information rapidly adapting action potential firing to a sustained deflection of only one whisker, deflection of other whiskers having no effect. This thus defines a singlewhisker receptive field for the trigeminal sensory neurons (Gibson and Welker 1983). Different sensory neurons respond with different thresholds of whisker movement and many are tuned to respond better to deflections in particular directions. The axons of these neurons terminate in clusters in the principal trigeminal nucleus of the brainstem (along with less-dense projections to three subdivisions of the spinal trigeminal nucleus). These clusters of axonal arborizations are arranged in a somatotopic pattern forming barrelettes that can be visualized by a cytochrome oxidase stain (Jacquin et al. 1993). The trigeminal neurons make excitatory glutamatergic synapses with the brainstem neurons. Since the dendritic fields of these neurons of the principal trigeminal nucleus are limited, they are innervated largely by the axonal arbors terminating in a single barrelette, in turn conveying the corresponding receptive field dominated by a single whisker (Chiaia et al. 1991; Lo et al. 1999; Veinante and Deschenes 1999). Neurons of the spinal trigeminal nuclei also receive sensory afferents, but most neurons will respond with action potential firing when any one of many whiskers is deflected, thus defining a multi-whisker receptive field (Jacquin and Rhoades 1990). Neurons in the principal trigeminal nucleus (with receptive fields dominated by a single whisker) form the so-called lemniscal sensory pathway, whereas neurons of the spinal trigeminal nucleus (with multi-whisker receptive fields) form the paralemniscal pathway. Ahissar et al. (2000) have suggested contrasting roles for these sensory pathways: the encoding of either spatial or temporal information. The ascending projections of these pathways remain largely separated with the spinal trigeminal neurons projecting to the posterior medial $(\mathrm{POm})$ nucleus of the thalamus and the neurons from principal nucleus projecting to the ventral posterior medial (VPM) nucleus of the thalamus. The axonal termination fields within the VPM are also segregated into somatotopically arranged clusters defining barreloids. The barreloid VPM neurons respond best to the aligned whisker, with some neurons having a singlewhisker receptive field, but surprisingly, in view of the precision of the anatomical barreloid wiring, many show multi-whisker receptive fields (Simons and Carvell 1989; Nicolelis et al. 1993; Brecht and Sakmann 2002a). Thalamic POm neurons are even more broadly tuned with large multi-whisker receptive fields (Diamond et al. 1992a) and respond with a long latency primarily during excitatory feedback from primary somatosensory cortex (Diamond et al. 1992b). Responses to single deflections of a single whisker are thus likely to be processed largely by activity in VPM neurons and primarily within the aligned principal whisker barreloid, whereas complex sensory stimuli involving many whiskers over prolonged periods of time may be processed equally by POm activity.

The VPM neurons project to the primary somatosensory cortex where they terminate in clusters within layer 4 of the neocortex, forming somatotopically organized barrels (Woolsey and Van der Loos 1970) along with a less-dense innervation of mid layer 5/6. The close correspondence of the pattern of layer 4 barrels with the layout of whiskers on the rodent snout is remarkable (Fig. 3). Not only is the structure beautiful, but it is also enormously useful to the investigator. When functional measurements are made of cortical activity they can be placed in the context of a well- 


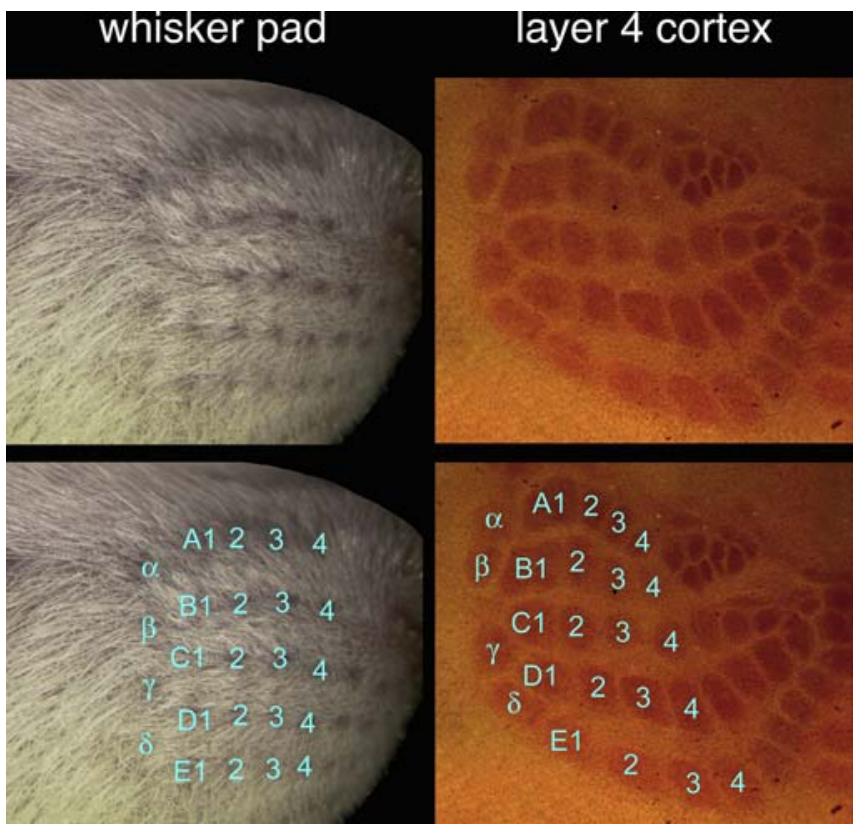

Fig. 3 Perhaps the most remarkable feature of the organization of the somatosensory cortex is the layout of the layer 4 barrels, which almost precisely matches the layout of the whiskers on the rodent snout. The tangential cortical layer 4 sections have been stained for cytochrome oxidase, which gives a clearly delineated pattern of barrels. Each of the larger barrels of the posterior whisker representation is around $400 \mu \mathrm{m}$ in diameter. Of especial use for physiological studies, the barrel pattern is also visible in bright field microscopy of living brain slices

defined anatomical map, which is important for quantitative analysis of response properties and essential for the study of cortical plasticity.

The POm neurons also project to primary somatosensory cortex, but with a pattern complementary to that in the VPM (Koralek et al. 1988). The POm afferents terminate in the layer 4 septa between barrels, as well as innervating both layers $2 / 3$ and $5 / 6$.

In addition the primary somatosensory cortex reciprocally innervates the thalamic nuclei, secondary somatosensory cortex, motor cortex and the contralateral somatosensory cortex. These higher-order feedback connections are likely to be of great importance during behaviour, but are as yet poorly understood. In the first steps to analyse cortical function quantitatively, simple stimuli have been used which will primarily signal through the lemniscal, labelled-line, single-whisker, anatomically-defined pathway of brainstem barrelettes, VPM barreloids and cortical processing beginning within the layer 4 barrels.

\section{The anatomical and functional organization of the barrel cortex}

The high degree of anatomical organization of the somatosensory neocortex immediately suggests a functional localization of neural activity related to each individual whisker. Since the whiskers operate in concert as a sensory organ, the exchange of information related to the individual whiskers is also likely to play a prominent role in cortical processing. One likely role for the barrel cortex is, then, to distribute the information related to the movement of a single whisker and compare this with information relating to movements of other whiskers. Such a process is likely to occur in a spatially and temporally distributed manner. Recently, the spatiotemporal dynamics of electrical activity resolved with millisecond precision and evoked by a single whisker deflection have been studied by two methods. One approach utilizes ordered arrays of extracellular electrodes recording action potential activity from a few cells in each barrel (Petersen and Diamond 2000). A complementary approach introduces voltage-sensitive dyes into the cortex. These dyes change their fluorescence depending upon membrane potential, and they can be imaged with $\sim 50 \mu \mathrm{m}$ spatial resolution and millisecond temporal resolution. They reveal primarily changes in ensemble subthreshold neuronal membrane potential (Petersen et al. 2003). These direct measurements of how cortical activity evoked by a single whisker is distributed spatiotemporally across the barrel cortex correlate well with measurements of receptive field properties of individual neurons analysed by sequentially deflecting many whiskers (Simons 1978; ArmstrongJames et al. 1992; Moore and Nelson 1998; Zhu and Connors 1999; Brecht and Sakmann 2002b; Petersen et al. 2003). The earliest sensory response occurs $\sim 8 \mathrm{~ms}$ following whisker deflection and is localized to the direct targets of the VPM input, the layer 4 barrel neurons and a fraction of neurons in mid layer 5/6. In the next milliseconds excitation propagates into layer $2 / 3$ in a columnar fashion. Thus a functional neocortical column bounded laterally by the layer 4 barrel structure is depolarized 10-12 ms after whisker deflection (Fig. 4). In the following milliseconds both infragranular neurons and neurons in neighbouring barrel columns become excited, apparently mainly through local cortical synaptic circuits. Excitation spreads preferentially along the row orientation of the barrel cortex, for example deflection of the D2 whisker evokes first a response in the D2 barrel column and over the next milliseconds the largest responses are found in D1 and D3 neighbouring barrel columns with smaller responses in the $\mathrm{C} 2$ or E2 columns. This oriented spread of excitation may serve a useful physiological function. The whisking behaviour involves rapid whisker movements oriented largely in a plane along the rows. Thus during the forward motion of the whiskers, the D3 whisker will pass through a point in space a few milliseconds before the D2 whisker, which in turn will be followed by the D1 whisker moving through the identical spatial location. Thus whiskers lying in the same row will often sample the same point in space within milliseconds of each other. For the animal to process this information relating to individual whiskers distributed across the neocortical barrel field, it is likely to be important that this single-whisker-related information is exchanged rapidly along the rows of the barrel cortex. The rapid spread of the sensory response may mediate this process, with propagation velocities along the row being twice as 
fast as along the orthogonal arc direction (Petersen et al. 2003).

These physiological responses to whisker stimulation recorded in vivo can now begin to be understood at the level of the synaptic interactions between neurons. An important step in this process has been the realization that the barrel field map, primarily visualized by histochemical stains can also be visualized in living brain slices (Petersen and Sakmann 2000). This allows neocortical circuitry to
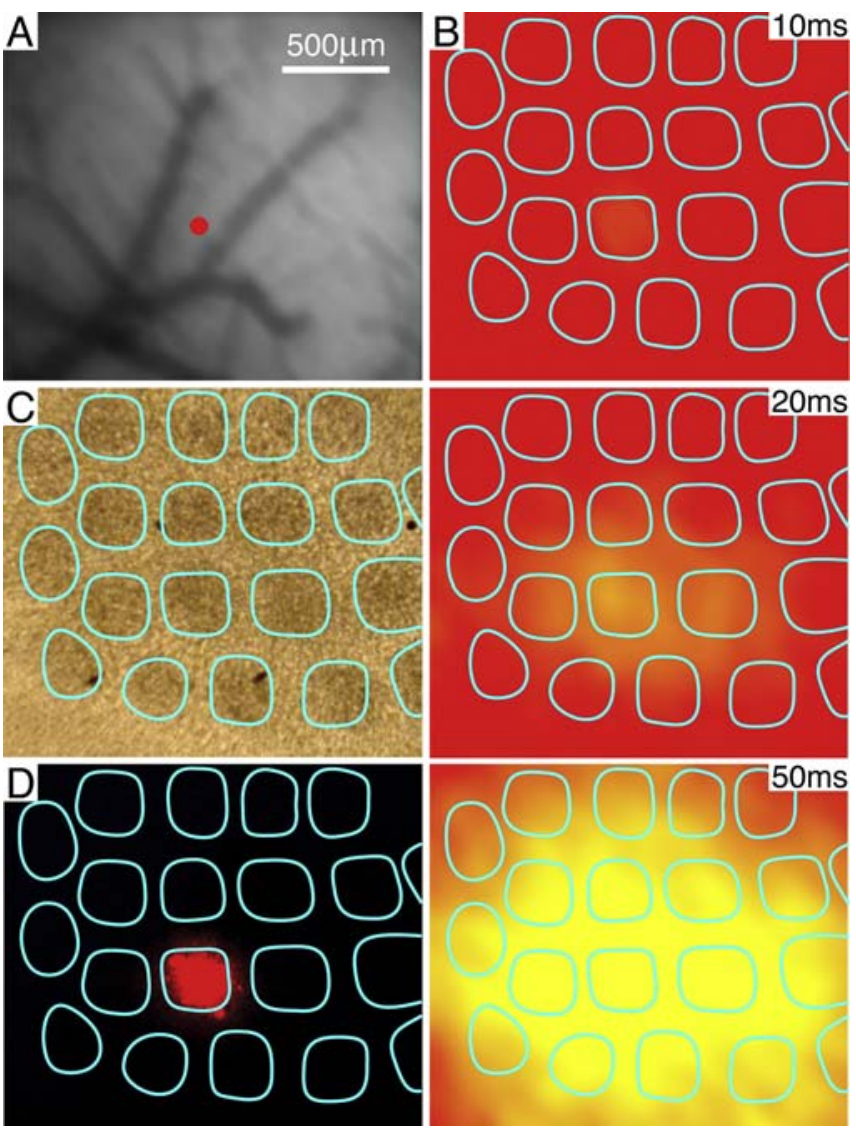

Fig. 4A-D Voltage-sensitive dyes can be used to map the spatiotemporal dynamics of the sensory response at millisecond temporal resolution and with a spatial resolution of $\sim 50 \mu \mathrm{m}$. A The cortical surface with the superficial blood vessels. The latter provide a spatial reference between post-mortem anatomical studies and in vivo studies. B Upper, middle and lower panels: time-dependent spread of subthreshold electrical activity in layer $2 / 3$ of the barrel cortex imaged with voltage-sensitive dye. The D2 whisker was deflected at time $0 \mathrm{~ms}$ for $2 \mathrm{~ms}$. The earliest cortical sensory response occurs $10 \mathrm{~ms}$ later in layer $2 / 3$ and is localized to the D2 barrel column (the yellow colour indicates depolarization). In the next tens of milliseconds, excitation propagates preferentially in a row-like manner and $50 \mathrm{~ms}$ after the whisker deflection the sensory response can be distributed almost evenly across the barrel field. After the imaging session 1,1'-dioctadecyl-3,3,3'3'-tetramethylindocarbocyanine perchlorate (DiI) was injected into the centre of the early response to compare the functional map with the anatomical map (the red dot in $\mathbf{A}$ indicates the injection site). The brain is subsequently sliced tangentially and, in $\mathbf{C}$, the layer 4 barrel field is outlined in cyan. The DiI fluorescence (D) is localized to the D2 barrel, indicating that the earliest sensory response originates from the appropriately aligned D2 barrel column. Modified and reprinted (with permission) from Petersen et al. (2003) Copyright: Society for Neuroscience be defined under controlled conditions by recording from pairs of anatomically identified neurons. Close agreement has been found between the functional synaptic connectivity and the density overlap of axonal and dendritic arbors, allowing qualitative and the beginnings of quantitative analysis of neocortical network connectivity. The first level of neocortical processing begins with the layer 4 barrel neurons, which are connected directly to thalamic VPM neurons through glutamatergic synapses (Agmon and Connors 1991). Both glutamatergic excitatory (spiny stellate and star pyramidal neurons) and diverse classes of GABAergic neurons in layer 4 receive direct VPM input (Porter et al. 2001; Bruno and Simons 2002). Excitatory layer 4 neurons within the same barrel are connected intimately, with approximately every third pair of neurons being synaptically connected, but there is very little synaptic connectivity between neighbouring barrels (Feldmeyer et al. 1999; Petersen and Sakmann 2000, 2001; Schubert et al. 2003; Shepard et al. 2003). As seen in Fig. 5, this pattern of connectivity probably results from the highly polarized dendritic and axonal arbors of these neurons, which rarely enter neighbouring layer 4 barrels (Lübke et al. 2000; Petersen and Sakmann 2000). Each layer 4 barrel therefore is an independent and irreducible unit consisting of a few thousand neurons which process information relating primarily to its isomorphic whisker. The ability to define the neuronal network both in terms of the number of participating neurons and the normal physiological input (since the location of the barrel in the sensory map can be established), makes the barrel cortex an ideal starting point for quantitative modelling of neocortical networks (Petersen 2002).

The excitatory layer 4 neurons project most densely into layer $2 / 3$ with the horizontal axonal field spreading little wider than the underlying layer 4 barrel, thus defining anatomically a neocortical column. Excitatory synaptic connections from layer 4 to layer $2 / 3$ pyramidal neurons occur frequently but have smaller efficacy and smaller NMDA receptor component than the layer 4 to layer 4 synapses (Feldmeyer et al. 2002). The flow of excitation is strictly feed forward since there are no reciprocal excitatory connections from layer $2 / 3$ to layer 4 . Layer $2 / 3$ pyramidal neurons synapse with their neighbouring layer $2 / 3$ pyramidal neurons, layer $5 / 6$ pyramidal neurons (Reyes and Sakmann 1999) and project to other cortical areas including contralateral somatosensory cortex, motor cortex and secondary somatosensory cortex). Within the local circuits their axonal fields do not respect barrel column boundaries (Fig. 5) extending far into neighbouring barrel columns. Synaptic excitation from these layer $2 / 3$ axons probably underlies the time-dependent spread of excitation across the barrel field evoked by a single whisker stimulus (Petersen et al. 2003). Indeed, the axonal field of individual layer $2 / 3$ pyramidal neurons is oriented preferentially along the rows of the barrel field, thus complementing the functional data showing the preferential spread of excitation along this cortical axis.

The activity of the excitatory neuronal network is likely to be strongly regulated by the many diverse types of 
A

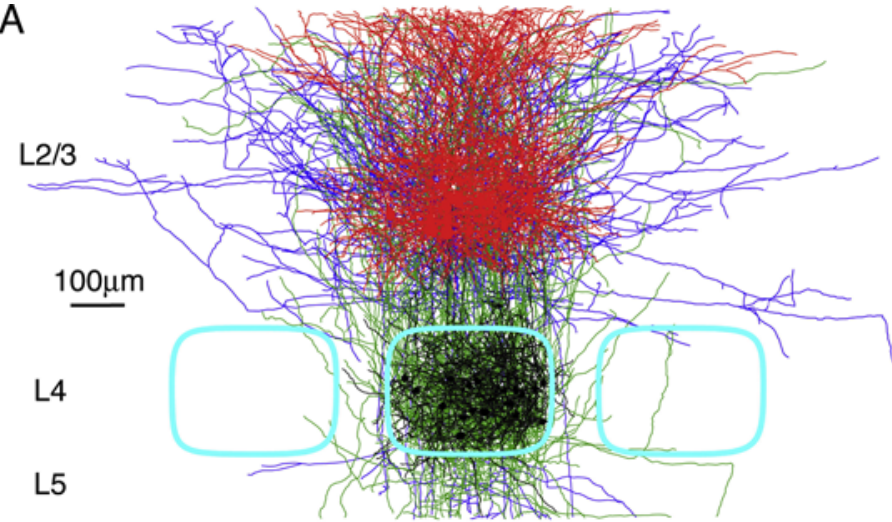

Fig. 5A, B Anatomical reconstructions of axons and dendrites of excitatory neurons in layers 2,3 and 4 in the context of the layer 4 barrels (outlined in cyan). Three-dimensional reconstructions were made from many neurons in different brain slices and normalized with respect to the barrel width and the depth of the layer $4 / 5$ boundary from the pia. This gives a representation of a normal barrel. In $\mathbf{A}$ all the structures are superimposed, in $\mathbf{B}$ the different compartments of each neuronal network are separated. The dendrites of layer $2 / 3$ pyramidal neurons are shown in red and their axons in blue. The dendrites of the layer 4 excitatory neurons are black whilst

cortical GABAergic interneurons (Gupta et al. 2000). These neurons receive strong excitatory synaptic input that can be distributed directly among coupled networks of interneurons through gap junctions, perhaps promoting highly precise action potential network synchrony (Galarreta and Hestrin 1999; Gibson et al. 1999).

Clearly, to understand the spatiotemporal dynamics of neocortical networks we need to measure from many neurons simultaneously and, to this end, imaging approaches have been applied utilizing either voltagesensitive or calcium-sensitive dyes. After staining entire neocortical slices or large areas of neocortex, voltagesensitive dyes measure subthreshold membrane potential changes (Petersen and Sakmann 2001) whereas calciumsensitive dyes primarily reveal action potential firing (Peterlin et al. 2000; Stosiek et al. 2003). Such network measurements have defined the functional architecture of the neocortical columns to superimpose on the anatomical barrel column of the layer 4 axonal field (Petersen and Sakmann 2001). At the cellular level, remarkably specific wiring patterns have been discovered in the relative locations of synaptically connected subtypes of neocortical neurons (Kozloski et al. 2001). An alternative optical technique for the analysis of neuronal circuits is to use light to stimulate neuronal activity, for example by uncaging glutamate (Callaway and Katz 1993). This elegant approach has been used to map the broad features of the barrel cortex circuitry (Schubert et al. 2003; Shepherd et al. 2003).

The technical advances in the optical probing of network activity will certainly give enormous additional insight, which for the barrel cortex can be integrated into the functional in vivo analysis of how these circuits are activated by sensory stimuli. A major research effort is therefore directed towards understanding quantitatively how barrel cortex neurons are excited by synaptic
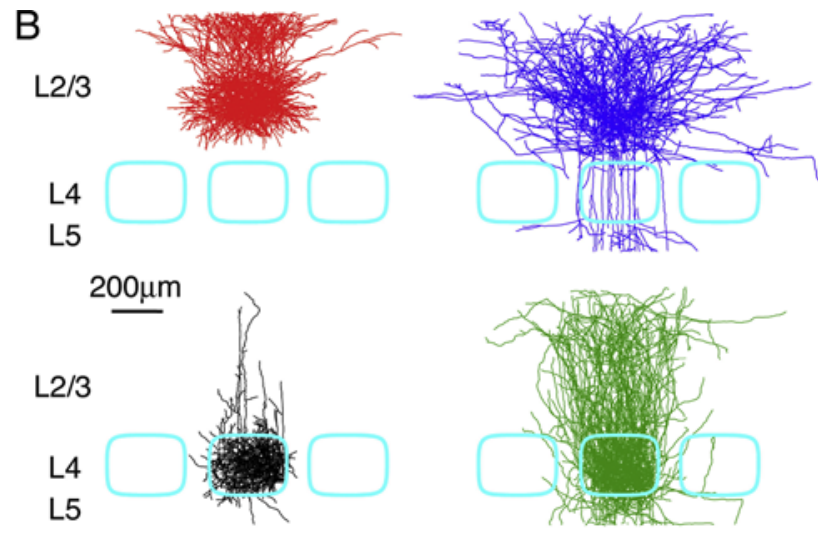

the axons of the latter are green. Across these different layers, it is obvious that the dendrites and axon of the layer 4 neurons are confined laterally to a barrel column and that the dendrites of the layer $2 / 3$ pyramidal neurons add only a little in width. Spread of excitation from one barrel column to the next will thus first take place at the level of layer $2 / 3$ action potential firing, when the long horizontal extent of these axons becomes relevant. Modified and reprinted (with permission) from Petersen and Sakmann (2001) Copyright: Society for Neuroscience

interactions to fire action potentials resulting from and interacting within a well-defined neuronal network. In addition to this challenge we must also understand both the developmental and the plasticity rules for wiring this cortical region, which will likely result from a complex interaction of genetics and experience.

\section{Development and plasticity of the barrel cortex}

Genetic determinants of somatotopic organization can be investigated with exquisite precision in the whisker-tobarrel cortex sensory pathway. Early studies have indicated that mice can be bred with either additional or fewer whiskers on their face and that the altered pattern of the sensory periphery was reflected fully by altered patterns of barrels in somatosensory cortex (Welker and Van der Loos 1986). More recently, ephrin gradients have been suggested to regulate the patterning of the barrel field and the size of barrels in different parts of the whisker representation (Vanderhaeghen et al. 2000). In a remarkable series of experiments, Fukuchi-Shimogori and Grove (2001) have demonstrated that manipulation of embryonic fibroblast growth factor (androgen-induced) (FGF8) expression can induce entire duplications of the barrel field. Thus, there is strong experimental evidence for genetic control in the patterning of barrel cortex. In addition early experiments have demonstrated a profound role for experience-dependent plasticity, since injury to whisker follicles eliminates the corresponding cortical barrels (Van der Loos and Woolsey 1973). The developmental time course of the whisker-pattern-related structures suggests that the periphery imposes structures on the higher levels of organization, beginning with brainstem barrelettes which are present at birth, later the thalamic barreloids and finally, by about post-natal day 5 , the neocortical layer 
4 barrels develop. Once the layer 4 barrel structure has formed it cannot be altered, so even damaging peripheral nerves leads to no alterations after a critical period during the first few days of life. However, although the largescale patterning of layer 4 does not remain plastic, many of the physiological and finer anatomical features of the barrel cortex can be changed in an experience-dependent way, even in adulthood. Thus the receptive fields can expand when all or all-but-one whiskers are trimmed (Simons and Land 1987; Glazewski and Fox 1996; Lendvai et al. 2000; Polley et al. 1999), but subtle differences in behaviour can reverse the plasticity into a contraction (Polley et al. 1999). To examine competition between the cortical representation of deprived and nondeprived whiskers, Diamond et al. (Diamond et al. 1994) introduced a pairing paradigm of two neighbouring intact whiskers whereby sensory responses are enhanced between the remaining whiskers. Thus at least two types of plasticity have been observed in barrel cortex: one that suggests expansion of non-deprived inputs into a deprived cortical area and a different form of plasticity that will wire regions of neocortex together that are processing similar sensory information. These alternative forms of plasticity are likely to result from different deprivation protocols; different developmental time points and differences in animal behaviour. The important conclusion is probably that there are multiple mechanisms for cortical plasticity and we must investigate each experimental paradigm carefully, in order first to understand at a cellular and synaptic level what plastic changes occur in an experiencedependent manner. Hopefully, we will then be able to identify and understand the basic rules for wiring the neocortex in an activity-dependent manner.

How these experience-dependent changes occur at the level of synaptic modifications in the cortical neuronal networks is beginning to be explored (Keller and Carlson 1999; Lendvai et al. 2000; Petersen et al. 2002; Allen et al. 2003; Shepherd et al. 2003). One important form of synaptic modification that may be involved is dependent upon activation of NMDA receptors (Bliss and Collingridge 1993) and occurs in a Hebbian manner during near synchronous action potential activity of the presynaptic and postsynaptic neurons (Markram et al. 1997; Sjöström et al. 2001). Such activity-dependent changes in the synaptic efficacy might occur continuously, in which case plasticity becomes closely entangled with neural coding, or, at the other extreme, plasticity might occur only during certain learning periods gated by the local brain state.

Which plastic changes occur are also likely to be regulated in part by genetic programs during development. It is likely that changes in gene expression close the critical period in thalamocortical plasticity (Crair and Malenka 1995), perhaps correlating with the critical period for large-scale remodelling of the barrel architecture. The genetic pathways involved in patterning the barrel field have been investigated using mainly transgenic mice. Such studies are seldom simple to interpret due to the complex interactions during development and multiple compensatory genes. Advances in targeting genetic alterations to specific brain regions in a temporally controlled fashion will likely reveal key elements in the specification of this sensory pathway. Recently mice with genetic alterations largely restricted to the cortex (Iwasato et al. 2000) have demonstrated conclusively that cortical NMDA receptors are involved in organising the structure of the neurons into a well-defined barrel field. Interestingly, in addition to this ionotropic receptor, a metabotropic glutamate receptor, signalling via phospholipase $\mathrm{C}$, has also been suggested to be involved specifically in the ordering the cortical layer 4 neurons into the barrel patterns (Hannan et al. 2001). A key goal now is to understand how these genetic manipulations are related to physiological alterations in neocortical function and ultimately to alterations in sensory perception.

\section{Perspectives}

The rodent barrel cortex with its well-defined somatotopic map offers immense experimental potential for studies concerning the structure and function of the neocortex integrated with quantitative behavioural analyses and defined molecular manipulations. Future work over the next decades will define the synaptic wiring diagram of the neocortical barrel column; how the network is physiologically activated by sensory input and the differential instructive roles of genetic programs and sensory experience. The ultimate goal of these studies is to understand the relationship of the brain to sensory perception. We clearly have much to learn, but the whisker-to-barrel cortex sensory system may allow these high-order questions to be approached for the first time with molecular and cellular resolution.

\section{References}

Agmon A, Connors BW (1991) Thalamocortical responses of mouse somatosensory (barrel) cortex in vitro. Neuroscience 41:365-379

Ahissar E, Sosnik R, Haiderliu S (2000) Transformation from temporal to rate coding in a somatosensory thalamocortical pathway. Nature 406:302-306

Allen CB, Celikel T, Feldman DE (2003) Long-term depression induced by sensory deprivation during cortical map plasticity in vivo. Nat Neurosci 6:291-299

Armstrong-James M, Fox K, Das-Gupta A (1992) Flow of excitation within rat barrel cortex on striking a single vibrissa. J Neurophysiol 68:1345-1358

Berg RW, Kleinfeld D (2003) Rhythmic whisking by rat: retraction as well as protraction of the vibrissae is under active muscular control. J Neurophysiol 89:104-117

Bliss TVP, Collingridge GL (1993) A synaptic model of memory: long-term potentiation in the hippocampus. Nature 361:31-39

Brecht M, Sakmann B (2002a) Whisker maps of neuronal subclasses of the rat ventral posterior medial thalamus, identified by whole-cell voltage recording and morphological reconstruction. J Physiol (Lond) 538:495-515

Brecht M, Sakmann B (2002b) Dynamic representation of whisker deflection by synaptic potentials in spiny stellate and pyramidal cells in the barrels and septa of layer 4 rat somatosensory cortex. J Physiol (Lond) 543:49-70 
Brecht M, Preilowski B, Merzenich MM (1997) Functional architecture of the mystacial vibrissae. Behav Brain Res 84:81-97

Bruno RM, Simons DJ (2002) Feedforward mechanisms of excitatory and inhibitory cortical receptive fields. J Neurosci 22:10966-10975

Callaway EM, Katz LC (1993) Photostimulation using caged glutamate reveals functional circuitry in living brain slices. Proc Natl Acad Sci USA 90:7661-7665

Carvell GE, Simons DJ (1990) Biometric analyses of vibrissal tactile discrimination in the rat. J Neurosci 10:2638-2648

Carvell GE, Simons DJ (1996) Abnormal tactile experience early in life disrupts active touch. J Neurosci 16:2750-2757

Chiaia NL, Rhoades RW, Bennett-Clarke CA, Fish SE, Killackey HP (1991) Thalamic processing of vibrissal information in the rat. I. Afferent input to the medial ventral posterior and posterior nuclei. J Comp Neurol 314:201-216

Crair MC, Malenka RC (1995) A critical period for long-term potentiation at thalamocortical synapses. Nature 375:325-328

Diamond ME, Armstrong-James M, Ebner FF (1992a) Somatic sensory responses in the rostral sector of the posterior group (POm) and in the ventral posterior medial nucleus (VPM) of the rat thalamus. J Comp Neurol 318:462-476

Diamond ME, Armstrong-James M, Budway MJ, Ebner FF (1992b) Somatic sensory responses in the rostral sector of the posterior group (POm) and in the ventral posterior medial nucleus (VPM) of the rat thalamus: dependence on the barrel field cortex. J Comp Neurol 319:66-84

Diamond ME, Huang W, Ebner FF (1994) Laminar comparison of somatosensory cortical plasticity. Science 265:1885-1888

Donoghue JP, Wise SP (1982) The motor cortex of the rat: cytoarchitecture and microstimulation mapping. J Comp Neurol 212:76-88

Dorfl J (1982) The musculature of the mystacial vibrissae of the white mouse. J Anat 135:147-154

Fee MS, Mitra PP, Kleinfeld D (1997) Central versus peripheral determinants of patterned spike activity in rat vibrissa cortex during whisking. J Neurophysiol 78:1144-1149

Feldmeyer D, Egger V, Lubke J, Sakmann B (1999) Reliable synaptic connections between pairs of excitatory layer 4 neurones within a single 'barrel of developing rat somatosensory cortex. J Physiol (Lond) 521:169-90

Feldmeyer D, Silver RA, Lübke J, Sakmann B (2002) Synaptic connections between layer 4 spiny neurone-layer $2 / 3$ pyramidal cell pairs in juvenile rat barrel cortex: physiology and anatomy of interlaminar signalling within a cortical column. J Physiol (Lond) 538:803-822

Fukuchi-Shimogori T, Grove EA (2001) Neocortex patterning by the secreted signaling molecule FGF8. Science 294:1071-1074

Galaretta M, Hestrin S (1999) A network of fast-spiking cells in the neocortex connected by electrical synapses. Nature 402:72-75

Gao P, Bermejo R, Zeigler HP (2001) Whisker deafferentation and rodent whisking patterns: behavioral evidence for a central pattern generator. J Neurosci 21:5374-5380

Gibson JM, Welker WI (1983) Quantitative studies of stimulus coding in first-order vibrissa afferents of rats. 1. Receptive field properties and threshold distributions. Somatosens Res 1:51-67

Gibson JR, Beierlein M, Connors BW (1999) Two networks of electrically coupled inhibitory neurons in neocortex. Nature 402:75-79

Glazewski S, Fox K (1996) Time course of experience-dependent synaptic potentiation and depression in barrel cortex of adolescent rats. J Neurophysiol 75:1714-1729

Gupta A, Wang Y, Markram H (2000) Organizing principles for a diversity of GABAergic interneurons and synapses in the neocortex. Science 287:273-278

Hannon AJ, Blakemore C, Katsnelson A, Vitalis T, Huber KM, Bear M, Roder J, Kim D, Shin H-S, Kind PC (2001) PLC- $\beta 1$, activated via mGluRs, mediates activity-dependent differentiation in cerebral cortex. Nat Neurosci 4:282-288
Harris JA, Petersen RS, Diamond ME (1999) Distribution of tactile learning and its neural basis. Proc Natl Acad Sci USA 96:75877591

Hattox AM, Priest CA, Keller A (2002) Functional circuitry involved in the regulation of whisker movements. J Comp Neurol 442:266-276

Hutson KA, Masterton RB (1986) The sensory contribution of a single vibrissas cortical barrel. J Neurophysiol 56:1196-1223

Iwasato T, Datwani A, Wolf AM, Nishiyama H, Taguchi Y, Tonegawa S, Knopfel T, Erzurumlu RS, Itohara S (2000) Cortex-restricted disruption of NMDAR1 impairs neuronal patterns in the barrel cortex. Neuron 406:726-731

Jacquin MF, Rhoades RW (1990) Cell structure and response properties in the trigeminal subnucleus oralis. Somatosens Mot Res 7:265-288

Jacquin MF, Renehan WE, Rhoades RW, Panneton WM (1993) Morphology and topography of identified primary afferents in trigeminal subnuclei principalis and oralis. J Neurophysiol 70:1911-1936

Keller A, Carlson GC (1999) Neonatal whisker clipping alters intracortical, but not thalamocortical projections, in rat barrel cortex. J Comp Neurol 412:83-94

Koralek K-A, Jensen KF, Killackey HP (1988) Evidence for two complementary patterns of thalamic input to the rat somatosensory cortex. Brain Res 463:346-351

Kozloski J, Hamzei-Sichani F, Yuste R (2001) Stereotyped position of local synaptic targets in neocortex. Science 293:868-872

Krupa DJ, Matell MS, Brisben AJ, Oliviera LM, Nicolelis MAL (2001) Behavioural properties of the trigeminal somatosensory system in rats performing whisker-dependent tactile discriminations. J Neurosci 21:5752-5763

Lendvai B, Stern EA, Chen B, Svoboda K (2000) Experiencedependent plasticity of dendritic spines in the developing rat barrel cortex in vivo. Nature 404:876-881

Lo F-S, Guido W, Erzurumlu RS (1999) Electrophysiological properties and synaptic responses of cells in the trigeminal principal sensory nucleus of postnatal rats. J Neurophysiol 82:2765-2775

Lübke J, Egger V, Sakmann B, Feldmeyer D (2000) Columnar organization of dendrites and axons of single and synaptically coupled excitatory spiny neurons in layer 4 of the rat barrel cortex. J Neurosci 20:5300-5311

Markram H, Lübke J, Frotscher M, Sakmann B (1997) Regulation of synaptic efficacy by coincidence of postsynaptic APs and EPSPs. Science 275:213-215

Miyashita E, Asanuma H, Keller A (1994) Input-output organization of the rat vibrissae motor cortex. Exp Brain Res 99:223-232

Moore CI, Nelson SB (1998) Spatio-temporal subthreshold receptive fields in the vibrissa representation of rat primary somatosensory cortex. J Neurophysiol 80:2882-2892

Nicolelis MAL, Lin RCS, Woodward DJ, Chapin JK (1993) Dynamics and distributed properties of many neuron ensembles in the ventral posterior medial thalamus of awake rats. Proc Natl Acad Sci USA 90:2212-2216

Peterlin ZA, Kozloski J, Mao BQ, Tsiola A, Yuste R (2000) Optical probing of neuronal circuits with calcium indicators. Proc Natl Acad Sci USA 97:3619-3624

Petersen CCH (2002) Short-term dynamics of synaptic transmission within the excitatory neuronal network of rat layer 4 barrel cortex. J Neurophysiol 87:2904-2914

Petersen CCH, Sakmann B (2000) The excitatory neuronal network of rat layer 4 barrel cortex. J Neurosci 20:7579-7586

Petersen CCH, Sakmann B (2001) Functionally independent columns of rat somatosensory barrel cortex revealed with voltage-sensitive dye imaging. J Neurosci 21:8435-8446

Petersen CCH, Brecht M, Sakmann B (2002) Activity-dependent synaptic wiring of layer $2 / 3$ rat barrel cortex underlying map plasticity. Program No. 413.3. 2002, Online Abstract Viewer/ Itinerary Planner, Society for Neuroscience, Washington, DC 
Petersen CCH, Grinvald A, Sakmann B (2003) Spatiotemporal dynamics of sensory responses in layer $2 / 3$ of rat barrel cortex measured in vivo by voltage-sensitive dye imaging combined with whole-cell voltage recordings and neuron reconstructions. J Neurosci 23:1298-1309

Petersen RS, Diamond ME (2000) Spatial-temporal distribution of whisker-evoked activity in rat somatosensory cortex and the coding of stimulus location. J Neurosci 20:6135-6143

Polley DB, Chen-Bee CH, Frostig RD (1999) Two directions of plasticity in the sensory-deprived adult cortex. Neuron 24:623637

Porter JT, Johnson CK, Agmon A (2001) Diverse types of interneurons generate thalamus-evoked feedforward inhibition in the mouse barrel cortex. J Neurosci 21:2699-2710

Prigg T, Goldreich D, Carvell GE, Simons DJ (2002) Texture discrimination and unit recordings in the rat whisker/barrel system. Physiol Behav 77:671-675

Reyes A, Sakmann B (1999) Developmental switch in the shortterm modification of unitary EPSPs evoked in layer $2 / 3$ and layer 5 pyramidal neurons of rat neocortex. J Neurosci 19:3827-3835

Schubert D, Kotter R, Zilles K, Luhmann HJ, Staiger JF (2003) Cell type-specific circuits of cortical layer IV spiny neurons. J Neurosci 23:2961-2970

Shepherd GM, Pologruto TA, Svoboda K (2003) Circuit analysis of experience-dependent plasticity in the developing rat barrel cortex. Neuron 38:277-289

Simons DJ (1978) Response properties of vibrissa units in rat SI somatosensory neocortex. J Neurophysiol 41:798-820

Simons DJ, Carvell GE (1989) Thalamocortical response transformation in the rat vibrissa/barrel system. J Neurophysiol $61: 311-330$
Simons DJ, Land PW (1987) Early experience of tactile stimulation influences organization of somatic sensory cortex. Nature 326:694-697

Sjöström PJ, Turrigiano GG, Nelson SB (2001) Rate, timing, and cooperativity jointly determine cortical synaptic plasticity. Neuron 32:1149-1164

Stosiek C, Garaschuk O, Holthoff K, Konnerth A (2003) In vivo two-photon calcium imaging of neuronal networks. Proc Natl Acad Sci USA 100:7319-7324

Vanderhaeghen P, Lu Q, Prakash N, Frisen J, Walsh CA, Frostig RD, Flanagan JG (2000) A mapping label required for normal scale of body representation in the cortex. Nat Neurosci 3:358365

Van der Loos H, Woolsey TA (1973) Somatosensory cortex: structural alterations following early injury to sense organs. Science 179:395-398

Veinante P, Deschenes M (1999) Single- and multi-whisker channels in the ascending projections from the principal trigeminal nucleus of the rat. J Neurosci 19:5085-5095

Welker WI (1964) Analysis of sniffing of the albino rat. Behaviour 22:223-244

Welker E, Van der Loos H (1986) Quantitative correlation between barrel-field size and the sensory innervation of the whiskerpad: a comparative study in six strains of mice bred for different patterns of mystacial vibrissae. J Neurosci 6: 3355-3373

Woolsey TA, Van der Loos H (1970) The structural organisation of layer IV in the somatosensory region (SI) of the mouse cerebral cortex: the description of a cortical field composed of discrete cytoarchitectonic units. Brain Res 17:205-242

Zhu JJ, Connors BW (1999) Intrinsic firing patterns and whiskersevoked synaptic responses of neurons in the rat barrel cortex. J Neurophysiol 81:1171-1183 